\title{
Ein Sandwich-Glyco-Immunoassay zur Detektion von Rizin in einem automatisierten Auslesesystem
}

\author{
Maria Hübner, Reinhard Nießner, Michael Seidel \\ Institut für Wasserchemie \& Chemische Balneologie, Lehrstuhl für Analytische Chemie, \\ Technische Universität München, Marchioninistr. 17, 81377 München, \\ Maria.Huebner@tum.de
}

\begin{abstract}
:
Rizin ist ein pflanzliches Toxin, das im Samen der Rizinus-Staude entsteht. Es gibt kein Gegengift zu Rizin und es wurde bereits als Biowaffe angewandt. Um es in einer wässrigen Probe schnell nachweisen zu können, wurde ein Sandwich Glyco-Immunoassay zur Detektion in einem automatisierten Auslesesystem entwickelt. Dabei wird die Eigenschaft von Rizin als Lektin genutzt. Es bindet mit hoher Affinität an D-Galactose-Reste auf Zelloberflächen. Deshalb werden D-Galactoseoder Lactose-Reste über Klick-Chemie mittels Kontaktdruck-Verfahren auf einer modifizierten GlasOberfläche immobilisiert. Für die Messung werden diese Kohlenhydrat-Mikroarrays in den Munich Chip Reader 3 (MCR 3) integriert. Dieses Gerät ermöglicht die automatische Durchführung des Sandwich-Glyco-Immunoassays und das Auslesen des Kohlenhydrat-Mikroarrays. Hierzu wird zunächst die wässrige Rizin-Probe über den Mikroarray geleitet. Anschließend wird ein Antikörper zugegeben, der gegen Rizin gerichtet ist und der mit Peroxidase markiert ist. Nach Zugabe der Substrate Wasserstoffperoxid und Luminol entsteht ein Chemilumineszenz-Signal, das über eine CCD-Kamera detektiert wird und mittels einer entsprechenden Software im angeschlossenen Computer ausgelesen werden kann. Das Signal kann ab einer Konzentration von $100 \mathrm{ng} \mathrm{mL}^{-1}$ Rizin detektiert werden, sodass tödliche Rizin-Dosen mit dem Sandwich Glyco-Immunoassay gemessen werden können.
\end{abstract}

Key words: Rizin, Kohlenhydrat-Mikroarray, Chemilumineszenz-Detektion, Sandwich-Glyco-Immunoassay

\section{Einleitung}

Rizin ist ein hochgiftiges Protein, das als Biowaffe verwendet werden kann.[1] Es kann durch Extraktion aus den Rizinussamen leicht gewonnen werden. Außerdem gibt es kein Gegengift und es kann potenziell dazu eingesetzt werden, Trinkwasser zu vergiften. Um rechtzeitig Gegen- und Schutzmaßnahmen einleiten zu können, werden automatisierte Screening-Methoden benötigt. Das Toxin selbst ist ein Protein mit einem Molekulargewicht von 60 - $65 \mathrm{kDa}$, sodass es auch als $\mathrm{RCA}_{60}$ bezeichnet wird. Es hat eine sogenannte A-BStruktur. Die A-Kette ist enzymatisch aktiv und für die Giftigkeit verantwortlich. Die B-Kette ist ein Lektin, das an Glycoproteine und Glycolipide auf Zelloberflächen binden kann, sodass es in die Zellen eindringen kann. In der vorgestellten Arbeit wird diese Lektin-
Untereinheit dazu benutzt, Rizin aus einer wässrigen Probe abzufangen und in einem automatisierten Chemilumineszenz-Auslesesystem zu quantifizieren.[2] Das Konzept des Sandwich - Glyco-Immunoassays ist in Abbildung 1 dargestellt. Die Rizin-Probe wird über den Glasträger geleitet, auf dem ein Saccharid immobilisiert wurde. Nach Zugabe eines mit Biotin modifizierten Antikörpers, der gegen die A-Kette des Rizin gerichtet ist, kann die Markierung mit einem Streptavidin-PeroxidaseKomplex erfolgen. Das ChemilumineszenzSignal (CL) kann in dem automatisierten Durchflusssystem MCR 3 durch Zugabe von Luminol und Wasserstoffperoxid erzeugt und ausgelesen werden.[3] Dieses Signal wird im MCR 3 von einer CCD-Kamera detektiert und mittels einer Software im angeschlossenen Computer ausgewertet. 

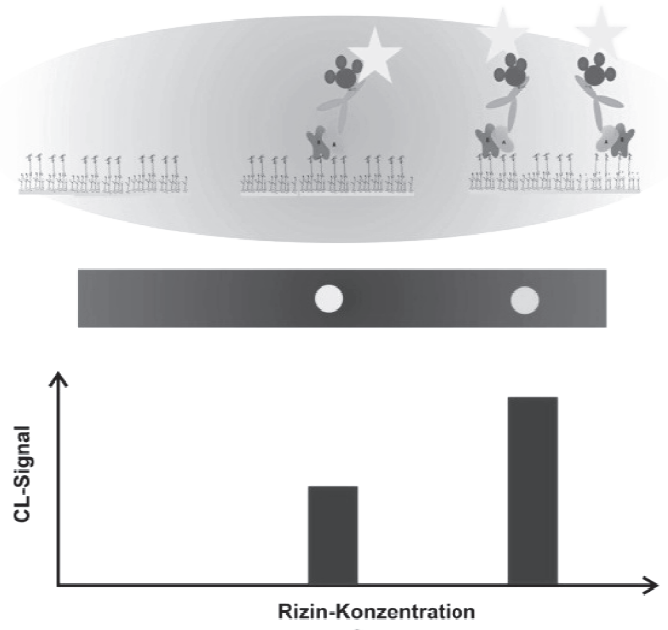

1.3

Biotinylierter monoklonaner Antikörper R 18

Streptavidin-Meerrettichperoxidase-Konjugat (Strep-polyHRP)

Chemilumineszenz (CL) Signal nach Zugabe von Luminol und $\mathrm{H}_{2} \mathrm{O}_{2}$

\section{Material und Methoden}

Die Glasträger wurden mit O-Proparglyoxy-Ntriethoxysilylpropylurethan (ABCR, Karlsruhe) belegt, sodass terminale Alkin-Gruppen auf der Oberfläche vorhanden waren. Diese wurden in einem Mikrokontakt-Drucker (Bio-Rad, München) mit dem 6-Azidohexyl-Lactose bzw. 6-Azidohexyl-Galactose modifiziert (Abbildung 2). Diese Klick-Reaktion konnte mit $2 \mathrm{mM}$ $\mathrm{CuSO}_{4}$-Lösung sowie $40 \mathrm{mM}$ Natriumascorbat in Wasser und 5\% Glyzerin durchgeführt werden. Vor dem Abchwaschen der Reaktionslösung mit Wasser wurden die Glasträger für $12 \mathrm{~h}$ bei $50 \%$ Luftfeuchtigkeit und $25^{\circ} \mathrm{C}$ inkubiert. Als Positiv-Kontrolle wurde Azide-PEG3-Biotin-Konjugat (Jena Bioscience, Jena) verwendet. Die so erhaltenen Kohlenhydrat-Mikroarrays wurden in die Flusszelle des MCR 3 integriert. Als Laufpuffer wurde PBST (10 mM KH $\mathrm{KOO}_{4}, 70 \mathrm{mM} \mathrm{K}_{2} \mathrm{HPO}_{4}$ und $145 \mathrm{mM} \mathrm{NaCl}, \quad 0,05 \% \mathrm{v} / \mathrm{w}$ Tween20) verwendet. Die Chemilumineszenz-Substrate
Abbildung 1: Konzept des Sandwich - GlycoImmunoassays zur Detektion von Rizin waren Luminol und $\mathrm{H}_{2} \mathrm{O}_{2}$ (Westar, Supernova, Bologna, Italien). Für die Assay-Entwicklung wurde anstatt Rizin $\left(\mathrm{RCA}_{60}\right)$, das weniger giftige Rizin Agglutinin $\left(\mathrm{RCA}_{120}\right)$ mit einer A-B-B-AStruktur verwendet. $\mathrm{RCA}_{60}$ wurde vom RobertKoch-Institut in Berlin bereit gestellt. $\mathrm{RCA}_{120}$ wurde von Sigma Aldrich (Steinheim) bezogen. Der Antikörper R-18-1 wurde ebenfalls vom Robert-Koch-Institut Berlin zur Verfügung gestellt. Der Munich Chip Reader 3 (MCR 3, GWK Präzisionstechnik, München) wird mit den entsprechenden Laufpuffer und Chemilumineszenz-Substraten ausgestattet. [3] $\mathrm{Er}$ wird durch das Programm LabVIEW 8.2 (National Instruments, Austin, USA) angesteuert. Die Chemilumineszenz-Signale werden mit Hilfe des MCR ImageAnalyzer Version 0.3.2.1 ausgewertet (GWK Präzisionstechnik, München). 

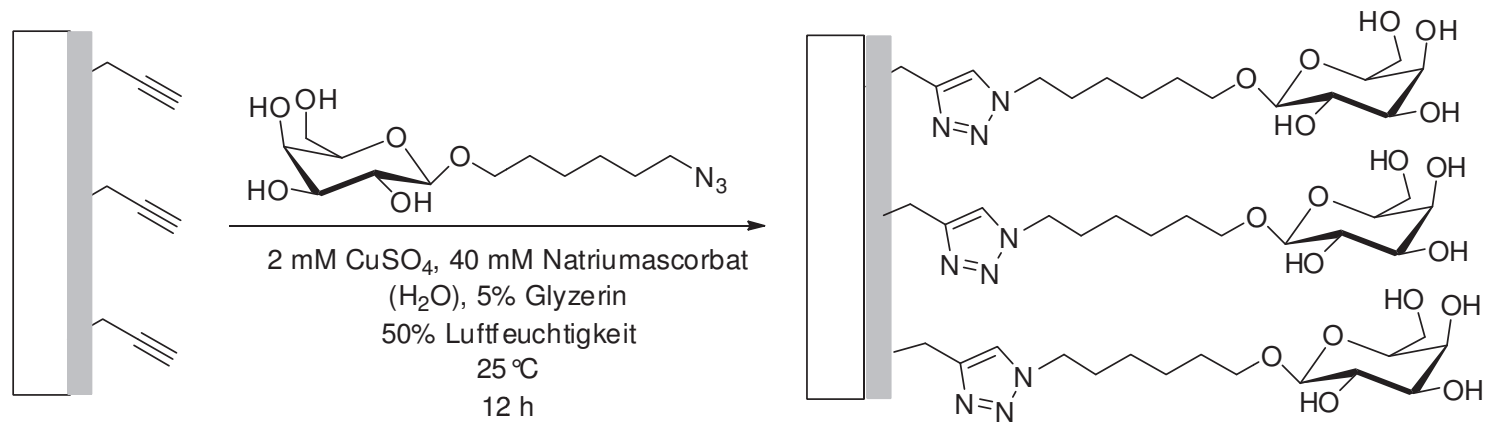

Abbildung 2: Immobilisierung des Saccharid-Derivates 6-Azidohexyl-D-Galactose auf einem modifizierten Glasträger mittels Mikrokontaktdruck-Verfahren.

\section{Ergebnisse}

Zunächst wurde getestet, welches Saccharid als Wechselwirkungspartner für RCA im Durchfluss geeignet ist (Abbildung 3 ). Es stellte sich heraus, dass Lactose im Vergleich zu Maltose und Glucose das höchste Signal liefert. Dieses Disaccharid trägt einen terminalen DGalactose-Rest. Sowohl Lactose als auch DGalactose können demnach als Wechselwirkungspartner für RCA verwendet werden.

Daraufhin wurde untersucht, wieviel Saccharid auf der Oberfläche benötigt wird, um RCA zu detektieren (Abbildung 4). Zur Detektion von $\mathrm{RCA}_{120}$ sollte eine mindestens $1 \mathrm{mM}$ DerivatLösung verwendet werden. Nach einer Zugabe von mindestens $5 \mathrm{mM}$ Saccharid-Lösung, ist

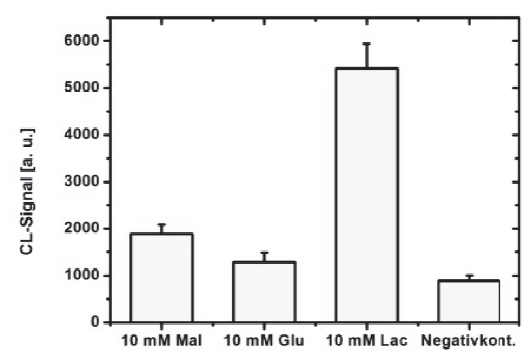

Abbildung 3: Vergleich verschiedener Kohlenhydrate (Mal: Maltose, Glu: Glucose, Lac: Lactose) zur Detektion von $R C A_{120}$. das maximale Signal erreicht. Für $\mathrm{RCA}_{60}$ ist das Signal im Allgemeinen höher. Die Bindung an die B-Untereinheit ist hier für die immobilisierten Saccharid-Reste ungehindert möglich. Mit steigender Saccharid-Konzentration nimmt das Chemilumineszenz-Signal zu, sodass durch die Saccharid-Dichte auf der Oberfläche Einfluss auf die Assay-Empfindlichkeit genommen werden kann. Die Assay-Empfindlichkeit wurde daraufhin für eine Saccharid-Konzentration von $10 \mathrm{mM}$ untersucht (Abbildung 5). Auf verschiedenen Glasträgern wurde das Chemilumineszenz-Signal in Abhängigkeit von der Rizin-Konzentration gemessen. Das Signal steigt bei $100 \mathrm{ng} \mathrm{mL}^{-1} \mathrm{RCA}_{60}$ an. Daher ist die Methode geeignet, eine tödliche Dosis in einem Glas Wasser zu detektieren (1-20 mg / kg Körpergewicht).[1]

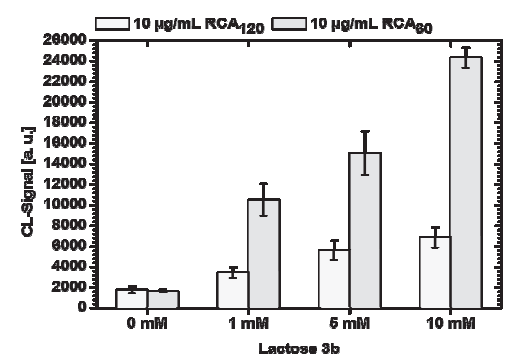

Abbildung 4: Abhängigkeit des ChemilumineszenzSignals von der Saccharid-Konzentration 


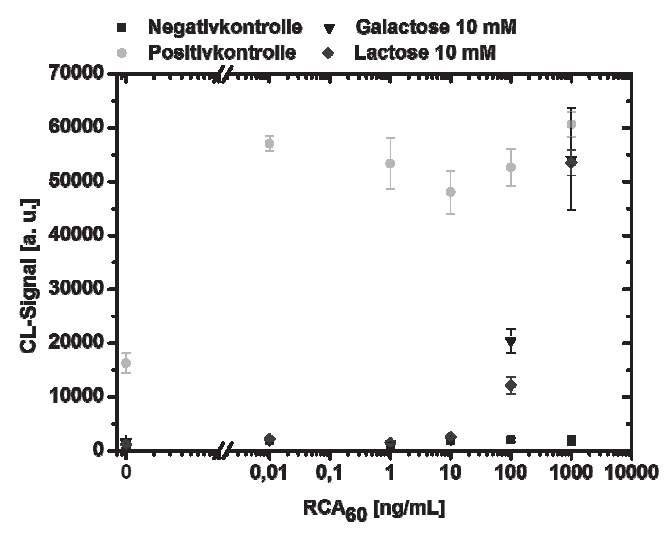

Abbildung 5: Konzentrationsabhängigkeit für den Sandwich Glyco-Immunoassay

\section{Ausblick}

In Zukunft kann das Prinzip eines SandwichGlyco-Immunoassays auf andere pflanzliche und bakterielle Toxine übertragen werden. Zum
Beispiel können die pflanzlichen Toxine Abrin, Viscumin, und Modeccin auch an D-Galactose gebunden werden. Ihre Unterscheidung wäre durch die Antikörpermarkierung möglich. Außerdem kann das Prinzip auch auf die bakteriellen Toxine wie Cholera-, Botulinum-, Tetanus- und Vero -Toxine übertragen werden. Folglich hat das hier vorgestellte Prinzip das Potenzial, als kostengünstige und vielseitige Screening-Methode für Biotoxine weiterentwickelt zu werden.

\section{Danksagung}

Die Autoren bedanken sich bei B. G. Dorner vom Zentrum für Biologische Sicherheit, Biologische Toxine (ZBS3), des Robert-KochInstituts Berlin für die freundliche Bereitstellung des $\mathrm{RCA}_{60}$ und des Antikörpers R-18-1.

\section{Referenzen}

[1] J. Audi, M. Belson, M. Patel, J. Schier, J. Osterloh, Ricin Poisoning: A Comprehensive Review, Journal of the American Medical Association 18, 2342-51 (2005); doi: 10.1001/jama.294.18.2342

[2] M. Huebner, K. Wutz, A. Szkola, R. Niessner, M. Seidel, A Glyco-Chip for the Detection of Ricin by an Automated Chemiluminescence Read-out System, Analytical Sciences 29, 461-466 (2013); doi: 10.2116/analsci.29.385

[3] K. Kloth, R. Niessner, M. Seidel, Development of an Open-Stand-Alone Platform for Regenerable Automated Microarrays, Biosensors \& Bioelectronics 24, 2106-2112 (2009); doi:

10.1016/j.bios.2008.11.005 